\title{
Intraoperative Molecular Analysis of Total Tumor Load in Sentinel Lymph Node: A Predictor of Axillary Status in Early Breast Cancer
}

\author{
Suphawat Laohawiriyakamol*, Somrit Mahattanobon, Puttisak Puttawibul
}

\begin{abstract}
Background: Axillary lymph node dissection (ALND) remains the standard of care in breast cancer patients with positive sentinel lymph node (SLN). However, approximately 40-60\% of patients with positive SLNs have not developed to non-SLN metastasis and ALND seems to be an overtreatment. The purpose of this study was to analyze predictors and define a specific cut-off of total tumor load (TTL) of CK19 that can be used as a predictive factor of non-SLN metastasis in early breast cancer patients. Materials and Methods: The records of 238 patients with cT1-3N0 breast cancer who had an intraoperative SLN evaluation performed through One-Step nucleic acid (OSNA) assay at Songklanagarind Hospital between 1 January 2015 and 31 December 2019 were examined. Univariate and Multivariate analysis was used to identify clinicopathologic features in SLN-positive patients that predict metastasis to non-SLNs. Finally, receiver operative characteristics (ROC) curves were used to choose an optimal TTL cut-off value. Results: Of a total of 110 patients who had a positive SLN, only 48 (43.64\%) were found to have positive nodes in non-SLN. Multivariate analysis revealed that lymphovascular invasion, type of SLN metastasis and SLN TTL (copies/ $\mu \mathrm{L}$ ) were independent predictors of positive non-SLNs. TTL cut-off value was 19,000 copies/ $\mu \mathrm{L}$, with an AUC of 0.838 with $72.7 \%$ sensitivity and $84.7 \%$ specificity to predict non-SLN metastasis. Conclusions: The likelihood of positive nonSLNs in patients who showed a positive SLN correlates with lymphovascular invasion, type of SLN metastasis and SLN TTL (copies $/ \mu L$ ). Our result revealed that the patients with a SLN TTL $\geq 19,000$ copies/ $\mu 1$ continue to attract the recommendation to proceed with ALND. This cut-off value can then help clinicians to assess which patients would benefit from ALND.
\end{abstract}

Keywords: Breast neoplasm - molecular diagnostic technique - sentinel lymph node biopsy - tumor load

Asian Pac J Cancer Prev, 23 (1), 349-354

\section{Introduction}

The diagnosis of lymph node metastasis is an important surgical procedure that will define the prognostics and treatment of breast cancer patients. Sentinel lymph node biopsy (SLNB) is routinely used for staging axillary lymph node status in clinical node negative breast cancer patients (Veronesi et al., 2006; Lyman et al., 2016). National Surgical Adjuvant Breast and Bowel Project (NSABP) trial B-32 showed that overall survival, disease-free survival, and regional control were not different between groups of SLN resection plus axillary lymph node dissection (ALND) and groups of SLN resection alone with ALND only if the SLNs were positive (Krag et al., 2010). Not all patients with positive SLNs have developed to non-SLN metastasis and ALND seems to be an overtreatment for these patients (Giuliano et al., 2011; Caudle et al., 2012; Laohawiriyakamol et al., 2017). The morbidity of ALND were more severe than SLNB, i.e. arm dysfunction, persistent pain, and edema of the upper arm lead to poor quality of life for those patients. Therefore, the discrimination of patients with positive SLNs who have no non-SLN metastasis is of very important clinical significance.

The One-Step nucleic acid amplification (OSNA, Sysmex, Kobe, Japan) method has been proposed as an effective tool for intraoperative detection of SLN macrometastasis, micrometastasis, and isolated tumor cells. OSNA method can evaluate a whole SLN by a reverse transcription loop-mediated isothermal amplification (RT-LAMP) of cytokeratin 19 (CK19) which is a duct epithelial cell marker in the SLN in a single reaction (Manzotti et al., 2001; Tsujimoto et al., 2007).

Total tumor load (TTL) which is total CK19 mRNA expression level in SLNs was used as a prediction tool of non-SLN metastasis in patients with early breast cancer and positive SLN. Cut-off values were defined to classify isolated tumor cells $(<250$ copies $/ \mu \mathrm{L})$, micrometastases 
(250-5,000 copies $/ \mu \mathrm{L})$, and macrometastases $(>5,000$ copies $/ \mu \mathrm{L}$ ) (Feldman et al., 2011; Tiernan et al., 2014). Previous studies revealed that $60.7 \%$ of the patients with macrometastatic SLN and $70.0 \%$ of patients with TTL in the SLN between 5,000 and 15,000 copies/ $\mu 1$ had no further nodal involvement on ALND (Peg et al., 2013; Fung et al., 2017). These results indicated that approximately half of SLN-positive patients undergo unnecessary ALND and this cut-off value might lead to overdiagnosis of SLN metastasis status. Until now, the optimal cut-off value of the TTL to predict the status of the NSLNs in patients with positive SLNB is still controversial.

The objective of this study was to determine whether the total tumor load (TTL) as indicated through the OneStep nucleic acid amplification (OSNA) assay can be a predictive factor of non-SLN metastasis in early breast cancer patients and define a specific cut-off of CK19 mRNA copy number.

\section{Materials and Methods}

The study design was approved by the Songklanagarind Hospital Ethics Committee. Informed consent was obtained from all patients.

\section{Patients}

The records of 238 patients with cT1-T3N0 breast cancer who had SLN analysis by OSNA assay at Songklanagarind Hospital between 1 January 2015 and 30 May 2016 were collected. The inclusion criteria included: patients with invasive breast cancer of cT1-T3, clinically N0, who have had SLN metastasis, and have undertaken ALND. The exclusion criteria included: patients who received systemic treatment either primary or neoadjuvant, ductal carcinoma in situ (DCIS), and local recurrence or systemic metastasis at the moment of diagnosis. The data collected from the medical records included age, SLN identification technique, size of SLN, tumor size, pathological T stage, histologic type, histologic grade, estrogen receptor (ER) and progesterone receptor (PR) status, human epidermal growth factor receptor 2 (HER2) status, the presence of lymphovascular invasion (LVI), type of SLN metastasis, total number of SLNs, number of positive and negative SLNs, and number of positive and negative non-SLNs. TTL was calculated as number of CK19 mRNA copies/ $\mu \mathrm{L}$ in the positive SLNs.

\section{SLN evaluation by OSNA assay}

SLN evaluation was performed by OSNA assay according to the manufacturer's instructions (Sysmex, Kobe, Japan). The OSNA assay for the detection of SLN metastasis was performed as previously described in detail (Tamaki et al., 2009; Hintzen et al., 2020). The results were reported according to the cut off level of calculated CK19 mRNA copies per $\mu \mathrm{L}$, non-metastasis was defined as $<250$ copies $/ \mu \mathrm{L}$, micrometastasis $(+)$ as $250-5,000$ copies $/ \mu \mathrm{L}$, and macrometastasis $(++)$ as $>5,000$ copies/ $\mu \mathrm{L}$ of CK19 mRNA. Mastectomy or lumpectomy was considered as part of the planned breast cancer treatment.

\section{Statistical analyses}

The Statistical Package for the Social Science (SPSS) for Windows version 23.0 (SPSS Inc., Chicago, IL, United States) was used for statistical analysis. The clinicopathological factors were compared between groups of patients with positive nodes in ALND and negative nodes in ALND. Categorical data were analyzed by Pearson's chi-square test. Continuous variables were analyzed by Mann-Whitney U-test. In SLN positive patients, univariate and multivariate logistic regression models were performed to assess the risk factors that were different between the non-SLN positive and negative groups with $\mathrm{p}$-value of $<0.05$ considered significant. The best cutoff of TTL was quantified with a receiver operating characteristics (ROC) curve as measured by the areas under receiver operating characteristic curves (AUC).

\section{Results}

\section{Patients'characteristics}

Of the 110 patients with positive SLNs evaluated by OSNA during surgery in this study, 48 (43.64\%) were found to have non-SLN metastasis while $62(56.36 \%)$ had no metastasis in non-SLN. A significant difference in age could be identified between patients with positive and negative nodes in non-SLN $(\mathrm{p}=0.03)$. In the group of patients with positive nodes in ALND, 8 patients $(16.7 \%)$ had a micrometastasis (OSNA+) and 40 patients (83.3\%) had a macrometastasis (OSNA++) in the SLN. On the other hand, of patients with no metastasis in ALND, 40 patients $(64.5 \%)$ had a micrometastasis (OSNA+) and 22 patients $(35.5 \%)$ had a macrometastasis (OSNA++) in the SLN. The mean TTL was 5,250 copies/ $\mu \mathrm{L}$ in patients with no metastasis in non-SLN and 19,870 copies/ $\mu \mathrm{L}$ in patients with metastasis in non-SLN. Characteristics of all patients are summarized in Table 1 .

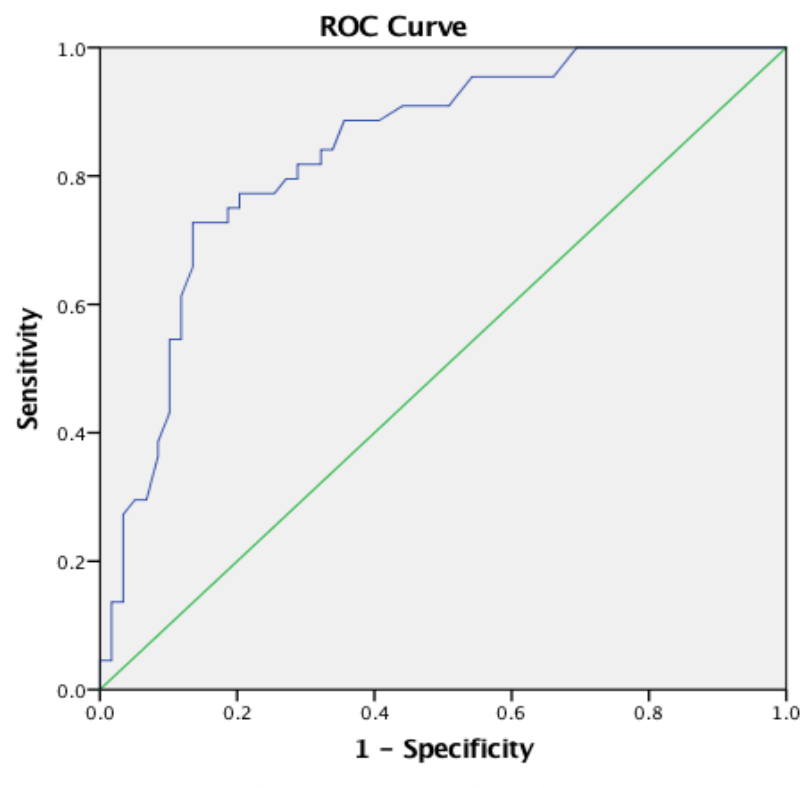

Diagonal segments are produced by ties.

Figure 1. Receiver Operative Characteristics (ROC) Curve Using TTL Variable as Predictor of non-SLN Metastasis (AUC $=0.838$, sensitivity $=72.7 \%$ and specificity $=84.7 \%$ ) 
Table 1. Patient and Tumors Characteristics Divided by Negative and Positive Non-SLNs

\begin{tabular}{|c|c|c|c|}
\hline \multirow[t]{2}{*}{ Characteristic } & \multicolumn{2}{|c|}{ Non-SLN in ALND } & \multirow[t]{2}{*}{$\mathrm{p}$ value } \\
\hline & Negative $(\mathrm{N}=62)$ & Positive $(\mathrm{N}=48)$ & \\
\hline \multicolumn{4}{|l|}{ Age (Year) } \\
\hline Mean & 54.7 & 50.5 & $0.03^{\mathrm{a}}$ \\
\hline$<45$ year $(\%)$ & $9(14.5)$ & $15(31.2)$ & $0.03^{b}$ \\
\hline$>=45$ year $(\%)$ & $53(85.5)$ & $33(68.8)$ & \\
\hline \multicolumn{4}{|l|}{ SLN identification } \\
\hline Dye alone & $13(21)$ & $10(20.8)$ & $0.98^{\mathrm{b}}$ \\
\hline Combine & $49(79)$ & $38(79.2)$ & \\
\hline Average SLNs (node) & 2.62 & 2.21 & $0.08^{a}$ \\
\hline Mean tumor size, $\mathrm{mm}$ & 2.21 & 2.66 & $0.06^{\mathrm{a}}$ \\
\hline \multicolumn{4}{|l|}{ Pathological T stage, no.(\%) } \\
\hline pT1a & $5(8.1)$ & $0(0)$ & $0.24^{b}$ \\
\hline pT1b & $5(8.1)$ & $3(6.2)$ & \\
\hline pT1c & $22(35.5)$ & $19(39.6)$ & \\
\hline pT2 & $30(48.3)$ & $25(52.2)$ & \\
\hline pT3 & $0(0)$ & $1(2)$ & \\
\hline \multicolumn{4}{|l|}{ Histologic type, no (\%) } \\
\hline Invasive ductal carcinoma & $61(98.4)$ & $45(93.8)$ & $0.09^{b}$ \\
\hline Invasive lobular carcinoma & $0(0)$ & $3(6.2)$ & \\
\hline Mucinous carcinoma & $1(1.6)$ & $0(0)$ & \\
\hline \multicolumn{4}{|l|}{ Histologic grade, no. (\%) } \\
\hline Grade I & $11(17.7)$ & $8(16.7)$ & $0.23^{\mathrm{b}}$ \\
\hline Grade II & $32(51.6)$ & $18(37.5)$ & \\
\hline Grade III & $19(30.7)$ & $22(45.8)$ & \\
\hline \multicolumn{4}{|l|}{ Estrogen receptor } \\
\hline Positive & $11(17.7)$ & $11(22.9)$ & $0.50^{\mathrm{b}}$ \\
\hline Negative & $51(82.3)$ & $37(77.1)$ & \\
\hline \multicolumn{4}{|l|}{ Progesterone receptor } \\
\hline Positive & $21(33.9)$ & $16(33.3)$ & $0.95^{\mathrm{b}}$ \\
\hline Negative & $41(66.1)$ & $32(66.7)$ & \\
\hline \multicolumn{4}{|l|}{ HER2 status } \\
\hline Positive & $44(71)$ & $37(77.1)$ & $0.47^{\mathrm{b}}$ \\
\hline Negative & $18(29)$ & $11(22.9)$ & \\
\hline \multicolumn{4}{|l|}{ Lymphovascular invasion } \\
\hline Present & $40(64.5)$ & $14(29.2)$ & $0.001^{\mathrm{b}}$ \\
\hline Absent & $22(35.5)$ & $34(70.8)$ & \\
\hline \multicolumn{4}{|l|}{ Type of SLN metastasis } \\
\hline Micrometastasis & $40(64.5)$ & $8(16.7)$ & $0.001^{\mathrm{b}}$ \\
\hline Macrometastasis & $22(35.5)$ & $40(83.3)$ & \\
\hline \multicolumn{4}{|l|}{ Number of Positive SLN } \\
\hline 1 & $5(8.1)$ & $0(0)$ & $0.24^{\mathrm{b}}$ \\
\hline 2 & $5(8.1)$ & $3(6.2)$ & \\
\hline 3 & $22(35.5)$ & $19(39.6)$ & \\
\hline 4 & $30(48.3)$ & $25(52.2)$ & \\
\hline 5 & $0(0)$ & $1(2)$ & \\
\hline Mean SLN TTL (copies/ $\mu \mathrm{L}$ ) & 5250 & 19870 & $0.001^{\mathrm{a}}$ \\
\hline \multicolumn{4}{|l|}{${ }^{a} p$ value by Mann-Whitney $U$ test } \\
\hline${ }^{\mathrm{b}} \mathrm{p}$ value by Chi-Square test & & & \\
\hline
\end{tabular}


Table 2. Univariable and Multivariable Associations of Prognostic Factors with Positive Non-SLN Metastasis

\begin{tabular}{|c|c|c|c|c|}
\hline & Odds ratio $(95 \% \mathrm{CI})$ & $\mathrm{p}$ value & Odds ratio $(95 \% \mathrm{CI})$ & $\mathrm{p}$ value \\
\hline Age, $<45$ yr vs. $>=45 \mathrm{yr}$ & $0.24(0.07 ; 0.86)$ & 0.02 & $0.25(0.07 ; 0.83)$ & 0.06 \\
\hline Identification, dye vs. combined & $0.78(0.23 ; 2.98)$ & 0.78 & & \\
\hline Average SLNs & $0.72(0.43 ; 1.21)$ & 0.22 & & \\
\hline Tumor size & $1.03(0.51 ; 2.10)$ & 0.92 & & \\
\hline Histologic grade, III vs. I/II & $1.26(0.60 ; 2.63)$ & 0.54 & & \\
\hline Estrogen receptor, -ve vs. +ve & $0.85(0.14 ; 5.10)$ & 0.86 & & \\
\hline Progesterone receptor -ve vs. +ve & $1.04(0.23 ; 4.72)$ & 0.95 & & \\
\hline HER2 status -ve vs. +ve & $0.67(0.18 ; 2.53)$ & 0.1 & & \\
\hline Present lymphovascular invasion & $3.81(1.51 ; 9.64)$ & 0.005 & $2.95(1.10 ; 7.88)$ & 0.031 \\
\hline SLN micro vs. macrometastasis & $8.33(2.72 ; 25.46)$ & 0.03 & $8.28(2.78 ; 24.65)$ & 0.001 \\
\hline Number of Positive SLN & $1.97(0.90 ; 4.30)$ & 0.08 & & \\
\hline SLN TTL (copies $/ \mu \mathrm{L})$ & $5.53(1.65 ; 18.43)$ & 0.005 & $5.48(1.72 ; 17.95)$ & 0.001 \\
\hline
\end{tabular}

SLN, sentinel lymph node; HER2, human epidermal growth factor receptor 2; TTL, total tumor load

Univariate and multivariate analysis of non-SLN metastasis

Results of the univariate and multivariate analyses are presented in Table 2. In the univariate analysis, patient age was found to have a statistically significant effect on non-SLN metastasis. Patients with age less than 45 years had a lower probability of positive non-SLN metastasis $(\mathrm{OR}=0.24,95 \% \mathrm{CI} ; 0.07-0.86, \mathrm{p}$ value $=$ 0.03 ). Second, we found a positive effect of the status of lymphovascular invasion, the risk for non-SLN metastasis increased when patients had lymphovascular invasion $(\mathrm{OR}=3.81,95 \% \mathrm{CI} ; 1.51-9.64, \mathrm{p}$ value $=0.005)$ According to type of SLN metastasis, macrometastasis in the SLN had a statistically significant association with non-SLN metastasis in the univariate analysis (OR, 8.33; 95\% CI, 2.75-25.46; p value 0.03 ) and in the multivariate analysis (OR, 8.28; 95\% CI, 2.78-24.65; p value 0.001). Subsequently, multivariate analyses were carried out on those variables found to be statistically significant on univariate analyses. Patient age displayed no difference in the multivariate analysis $(\mathrm{OR}=0.25,95 \% \mathrm{CI}$; $0.07-0.83$, $\mathrm{p}$ value $=0.06)$ whereas, lymphovascular invasion, type of SLN metastasis and SLN TTL (copies/ $\mu$ L) showed a significant association with the incidence of non-SLN metastases.

Receiver operating characteristic curve demonstrating diagnostic accuracy

Receiver operative characteristics (ROC) curves were then used to choose an optimal cut-off value with the highest sensitivity and specificity. Results from ROC analyses, with an AUC of 0.838 , identified a cut-off equal to 19,000 copies/mL showing $72.7 \%$ sensitivity and $84.7 \%$ specificity as in Figure 1.

\section{Discussion}

SLNB is routinely used for staging axillary lymph node status of early breast cancer patients (Veronesi et al., 2006; Lyman et al., 2016). If any SLNs contain cancer cells, ALND will be needed. However, in our study, of 110 patients with positive SLNs, only 48 (43.64\%) patients showed positive nodes in non-SLN. Our result is consistent with previous studies in that $\sim 50 \%$ of SLN-positive patients had non-SLN metastasis (Peg et al., 2013; Fung et al., 2017). Therefore, for approximately half of SLN-positive patients it was not always necessary to perform ALND and the prediction of non-SLN metastasis status based on whether metastasis was positive or negative in SLNs was insufficient. In this study, we tried to analyzed predictors and define a specific cut-off of TTL to reduce the overtreatment in SLN-positive patients.

Several previous studies have analyzed and developed predictors for non-SLN metastasis in patients with positive SLNs. In our study both of the univariate and multivariate analysis, lymphovascular invasion (LVI) status, type of SLN metastasis and SLN TTL (copies/ $\mu \mathrm{L}$ ) were significantly associated with non-SLN metastasis, consistent with the majority of other research (Hwang et al., 2003; Van zee et al., 2003; Osako et al., 2013; Nadeem et al., 2014; Teramoto et al., 2014). LVI has been described as the strongest independent predictor of nodal involvement. We found that, LVI was a significant predictor of non-SLN involvement; more than $70 \%$ of our patients with LVI had positive non-SLN and seemed to show higher odds (OR, 2.95; 95\% CI, 1.10-7.88; p value 0.031 ) as compared with patients with negative non-SLN. Whereas patients with macrometastasis had higher odds ratio (OR, 8.28; 95\% CI, 2.78-24.65; $p$ value 0.001 ) than micrometastasis.

CK19 is part of the cytoskeleton of epithelial cells and is normally not expressed in lymphatic tissue. Many investigators have suggested that the total CK19 mRNA copy number of SLN could be an important factor in predicting non-SLN metastasis. Currently, the molecular detection of CK19 by OSNA assay is a routine method that can be completed intraoperatively within 30-40 minutes (Tamaki et al., 2009; Peg et al., 2013; Fung et al., 2017; Hintzen et al., 2020). Cutoff values defined SLN as negative nodes when there are less than 250 copies/ $\mu \mathrm{L}$, micrometastasis if there are more than 250 copies $/ \mathrm{mL}$ but less than 5,000 copies/mL and macrometastases if there are more than 5,000 copies $/ \mu \mathrm{L}$ as reported from Feldman et al., (2011) and Tiernan et al., (2014) 
In our result, the group of patients with negative nodes in non-SLN showed mean SLN TTL 5,250 copies/ $\mu \mathrm{L}$ and patients with positive nodes in non-SLN showed mean SLN TTL 19,870 copies/ $\mu \mathrm{L}$. Results from our ROC analyses, with an AUC of 0.838 , identified a best TTL cut-off equal to 19,000 copies/mL (Sensitivity $72.7 \%$, Specificity $84.7 \%$ ) in predicting non-SLN metastasis in our cohort. Numerous studies have reported the results of the cut-off levels of CK19 copy number predict nonSLN metastasis, Recently, Nabias et al., (2017) proposed the TTL cut- off level at 190,000 copies/ $\mu 1$ with $73.3 \%$ sensitivity and $74.4 \%$ specificity. This study provided a rather high TTL cut- off level, however their sample size was quite small. In addition, Espinosa-Bravo et al., (2013) suggested 12,000 copies/ $\mu \mathrm{L}$ with a specificity of $85.3 \%$ and negative predictive value (NPV) of $80 \%$ in their cohort of patients. Terrenato et al., (2017) found a TTL cutoff $>2150$ copies $/ \mu \mathrm{L}$ with $95 \%$ sensitivity but the specificity was only $51 \%$. Deambrogio et al., (2014) identified the TTL cut- off at 7,700 copies/ $\mu \mathrm{L}$ with $78 \%$ sensitivity and 57\% specificity and Peg et al., (2013) suggested TTL cutoff point of 5,000 copies/ $\mu \mathrm{L}$ with $76.7 \%$ sensitivity and $55.2 \%$ specificity. TTL cut-off value from these studies remain inconsistent and suggests that the same cut-off value cannot be applied to other populations. The reason possibly may be due to different in size of axillary lymph node, variations of demographics and locations of each patient population. With our new recommended threshold, patients with TTL less than 19,000 copies/mL had no non-SLN metastasis and no need to recieve an ALND.

In summary, our study demonstrated that only $43.64 \%$ of patients with positive SLNs showed positive nodes in non-SLN. LVI status, type of SLN metastasis and SLN TTL (copies $/ \mu \mathrm{L}$ ) were independent predictors of non- SLN metastases. Furthermore, we propose that, the cutoff level at 19,000 copies/ $\mu$ lis the best cutoff with sensitivity $72.7 \%$ and specificity $84.7 \%$ in predicting non-SLN metastasis in our cohort.

\section{Author Contribution Statement}

This study was designed, provided conceptual, directed, and coordinated by Laohawiriyakamol S and Puttawibul P. The data were collected and analyzed by all authors. The manuscript was written by Laohawiriyakamol $\mathrm{S}$ and commented on by all authors.

\section{Acknowledgements}

We thank all members of the Division of Surgery at Faculty of Medicine, Prince of Songkla University for their contribution to the parts of the medical record service. We thank the International Affairs, Faculty of Medicine, Prince of Songkla University, for help in the English language editing of the manuscript. Special thanks also to all patients who participated in the data collection process.

\section{Funding}

The study received a research grant from Faculty of Medicine, Prince of Songkla University
Molecular Analysis of Sentinel Lymph Node in Breast Cancer

Ethical approval

The study was approved by the Institutional Review Board of Faculty of Medicine, Prince of Songkla University (No.58-035-10-1)

\section{Availability of data}

The datasets are not publicly available due to ethical restrictions, but are available from the corresponding author on reasonable request.

\section{Conflict of Interest}

all authors declare no conflict of interest in this study.

\section{References}

Caudle AS, Hunt KK, Tucker SL, et al (2012). American college of surgeons oncology group (ACOSOG) Z0011: impact on surgeon practice patterns. Ann Surg Oncol, 19, 3144-51.

Deambrogio C, Castellano I, Paganotti A, et al (2014). A new clinical cut-off of cytokeratin 19 mRNA copy number in sentinel lymph node better identifies patients eligible for axillary lymph node dissection in breast cancer. $J$ Clin Pathol, 67, 702-6.

Espinosa-Bravo M, Sansano I, Pérez-Hoyos S, et al (2013). Prediction of non-sentinel lymph node metastasis in early breast cancer by assessing total tumoral load in the sentinel lymph node by molecular assay. EJSO, 39, 766-73.

Feldman S, Krishnamurthy S, Gillanders W, et al (2011). A novel automated assay for the rapid identification of metastatic breast carcinoma in sentinel lymph nodes. Cancer, 117, 2599-607.

Fung V, Kohlhardt S, Vergani P, et al (2017). Intraoperative prediction of the two axillary lymph node macrometastases threshold in patients with breast cancer using a one-step nucleic acid cytokeratin-19 amplification assay. Mol Clin Oncol, 7, 755-62.

Giuliano AE, Hunt KK, Ballman KV, et al (2011). Axillary dissection vs no axillary dissection in women with invasive breast cancer and sentinel node metastasis: A Randomized Clinical Trial. JAMA, 305, 569-75.

Hintzen KFH, Rooij LD, Schouten N, et al (2020). Molecular analysis of sentinel lymph nodes in patients with breast cancer using one-step nucleic acid amplification (OSNA): Does not lead to overtreatment in the current era of deescalating axillary management. Surg Oncol, 35, 224-8.

Hwang RF, Krishnamurthy S, Hunt KK, et al (2003). Clinicopathologic factors predicting involvement of nonsentinel axillary nodes in women with breast cancer. Ann Surg Oncol, 10, 248-54.

Krag DN, Anderson SJ, Julian TB, et al (2010). Sentinel-lymphnode resection compared with conventional axillary-lymphnode dissection in clinically node-negative patients with breast cancer: overall survival findings from the NSABP B-32 randomised phase 3 trial. Lancet Oncol, 11, 927-33.

Laohawiriyakamol S, Mahattanobon S, Puttawibul P (2017). The pre-treatment neutrophil-lymphocyte ratio: a Useful Tool in Predicting Non-Sentinel Lymph Node Metastasis in Breast Cancer Cases. Asian Pac J Cancer Prev, 18, 557-62.

Lyman GH, Somerfield MR, Bosserman LD, et al (2016). Sentinel lymph node biopsy for patients with early-stage breast cancer: American Society of Clinical Oncology Clinical Practice Guideline Update. J Clin Oncol, 35, 561-4.

Manzotti M, Dell'Orto P, Maisonneuve P, et al (2001). Reverse transcription polymerase chain reaction assay for multiple mRNA markers in the detection of breast cancer metastases in sentinel lymph nodes. Int J Cancer, 95, 307-12.

Asian Pacific Journal of Cancer Prevention, Vol 23 
Nabais C, Figueiredo J, Lopes P, et al (2017). Total tumor load assessed by one-step nucleic acid amplification assay as an intraoperative predictor for non-sentinel lymph node metastasis in breast cancer. Breast J, 32, 33-6.

Nadeem RM, Gudur LD, Saidan ZA (2014). An independent assessment of the 7 nomograms for predicting the probability of additional axillary nodal metastases after positive sentinel lymph node biopsy in a cohort of British patients with breast cancer. Clin Breast Cancer, 14, 272-9.

Osako T, Iwase T, Kimura K, et al (2013). Sentinel node tumour burden quantified based on cytokeratin 19 mRNA copy number predicts non-sentinel node metastases in breast cancer: Molecular whole-node analysis of all removed nodes. Eur J Cancer, 49, 1187-95.

Peg V, Espinosa-Bravo M, Vieites B, et al (2013). Intraoperative molecular analysis of total tumor load in sentinel lymph node: A new predictor of axillary status in early breast cancer patients. Breast Cancer Res Treat, 139, 87-93.

Tamaki Y, Akiyama F, Iwase T, et al (2009). Molecular detection of lymph node metastases in breast cancer patients: Results of a Multicenter Trial Using the One-Step Nucleic Acid Amplification Assay. Clin Cancer Res, 15, 2879-84.

Teramoto A, Shimazu K, Naoi Y, et al (2014). One-step nucleic acid amplification assay for intraoperative prediction of non-sentinel lymph node metastasis in breast cancer patients with sentinel lymph node metastasis. Breast J, 23, 579-85.

Terrenato I, D'Alicandro V, Casini B, et al (2017). A cut-off of 2150 cytokeratin 19 mRNA copy number in sentinel lymph node may be a powerful predictor of non-sentinel lymph node status in breast cancer patients. PLoS One, 12, e0171517.

Tiernan J, Verghese E, Nair A, et al (2014). Systematic review and meta-analysis of cytokeratin 19-based one-step nucleic acid amplification versus histopathology for sentinel lymph node assessment in breast cancer. Br J Surg, 101, 298-306.

Tsujimoto M, Nakabayashi K, Yoshidome K, et al (2007). Onestep nucleic acid amplification for intraoperative detection of lymph node metastasis in breast cancer patients. Clin Cancer Res, 13, 4807-16.

Van Zee KJ, Manasseh D-ME, Bevilacqua JLB, et al (2003). A nomogram for predicting the likelihood of additional nodal metastases in breast cancer patients with a positive sentinel node biopsy. Ann Surg Oncol, 10, 1140-51.

Veronesi U, Paganelli G, Viale G, et al (2006). Sentinel-lymphnode biopsy as a staging procedure in breast cancer: update of a randomised controlled study. Lancet Oncol, 7, 983-90.

\section{@()}

This work is licensed under a Creative Commons AttributionNon Commercial 4.0 International License. 